\title{
Stability and bifurcation of planetary dynamo models
}

\author{
E. Dormy $\dagger$
}

CNRS \& MAG (ENS/IPGP), Département de Physique, Ecole Normale Supérieure, 24 rue Lhomond, 75252 Paris CEDEX 05, France

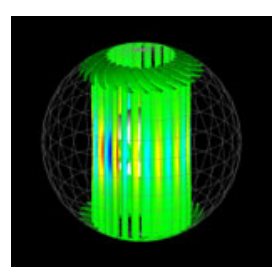

Rapidly rotating dynamos, relevant to the origin of the Earth magnetic field, are difficult to model owing to the extreme parameter regimes that occur in their dynamics. Numerical models alone fail to approach the correct regime. However, progress can be achieved by combining numerical and analytical methods. This can offer a better understanding of the variety of behaviour observed near the onset of dynamo action, as seen in the recent study of Sreenivasan \& Jones (J. Fluid Mech., this issue, vol. 688, 2011, pp. 5-30).

\section{Introduction}

The origin of planetary magnetic fields, and in particular that of our own planet, the Earth, is a longstanding open issue. Self-excited dynamo action is thought to operate some $3000 \mathrm{~km}$ below our feet in the liquid core of the Earth. Dynamo theory involves a transfer of kinetic energy of a conducting liquid to magnetic energy through the amplification of electrical currents. 'Self-excited' implies that this transfer takes the form of an instability. Past a given threshold, some flows can amplify a seed magnetic field, against ohmic dissipation. Understanding the precise conditions under which this transfer of energy can occur constitutes an active area of research. Apart from very particular flows, which can be tackled analytically, numerical simulation is the only way to establish whether or not a flow can achieve this transition.

Current computational models cannot reach the extreme parameters needed for dynamo action in liquid metals. The ratio of the viscosity to the magnetic diffusivity, the magnetic Prandtl number, is less than $10^{-5}$ for all liquid metals. For magnetic field amplification to take place, the magnetic Reynolds number, $R m \equiv U L / \eta$, where $\eta$ is the ohmic diffusivity, needs to be large enough, typically of order 100 or larger. The ratio of the classical Reynolds number $(R e \equiv U L / v)$ to the magnetic Reynolds number is precisely the magnetic Prandtl number $(P m \equiv v / \eta)$. As a result the Reynolds number corresponding to dynamo action in liquid metals always exceeds $10^{6}$. Current numerical resources cannot reach such extreme values, and the magnetic Prandtl number is therefore overestimated by several orders of magnitude in all current numerical models.

The issue gets even worse when considering planetary dynamos, and in particular the geodynamo. The Earth rotates once a day, but the secular evolution of the

$†$ Email address for correspondence: dormy@phys.ens.fr 
geomagnetic field takes hundreds of years. The dimensionless parameter measuring this ratio, the Rossby number, is therefore very small. The Ekman number, which measures the ratio of the rotation period to the viscous diffusion time scale, $E=v / \Omega L^{2}$, is again a very small parameter. For the Earth, $E \sim 10^{-14}$, and this number is again always overestimated by factors of $10^{6}$, if not $10^{9}$, in current models. Such extreme parameters cause serious difficulties for numerical modelling. However, they provide a solid basis for asymptotic analysis. Expansions can be performed on the basis of small parameters, and exact analytical solutions can sometimes be exhibited for simplified linearized problems.

Even in such simplified configurations, the analytical work is often difficult. Indeed, a correct understanding of the onset of convection in a rapidly rotating sphere required many studies over a long period of time (Roberts 1965, 1968; Busse 1970; Yano 1992; Jones, Soward \& Mussa 2000; Dormy et al. 2004). The two complementary means of investigation offered by analytical and numerical work therefore unfortunately seldom interact, as they cover very different parameter regimes. To offer a simple summary, one may say that asymptotic expansions offer exact solutions for simplified problems, whereas numerical work addresses the relevant problem, but not in a suitable parameter regime. Links between both approaches are very rare. It is however clear that progress can only be achieved by trying to combine and compare these two means of investigation. Such is the case for the new study presented by Sreenivasan $\&$ Jones (2011).

\section{Overview}

Dynamo action can occur at large enough energy input, usually measured by the Rayleigh number, but not necessarily for all types of flow. However, the onset of dynamo action can be complicated. The induction equation governing the evolution of magnetic field $\mathbf{B}$ is linear, so exponentially growing seed fields are possible. Two solutions always destabilize at the same critical Rayleigh number, $R a=R a_{c}$, corresponding to the two possible polarities of the same eigenmode. In the classic scenario, the bifurcation is supercritical (figure 1a). Conservation of energy acting through the Lorentz force ensures that the exponential growth is not unbounded past the threshold. The Lorentz force modifies the flow, choking off dynamo growth, and since the Lorentz force is quadratic in $\mathbf{B}$ its effects on the flow are identical for both parities.

If the leading order effects of the Lorentz force are to saturate the growth of the magnetic field, the bifurcation is supercritical, and a typical graph of the magnetic field amplitude versus the control parameter is given in figure 1(a). If, however, the leading order effects of the Lorentz force are to destabilize the field further, saturation necessarily involves higher order effects and the bifurcation is then subcritical (as depicted in figure $1 b$ ). Over the last few years, several numerical dynamo models in rotating spherical shells revealed a hysteretic behaviour, characteristic of the latter type of bifurcation (Christensen, Olson \& Glatzmaier 1999; Morin 2005; Kuang, Jiang \& Wang 2008; Morin \& Dormy 2009). There were however no hints of the mechanisms by which the Lorentz force was to destabilize the magnetic field further.

Sreenivasan \& Jones (2011) were able to show, by combining direct numerical simulations and asymptotic developments on the linearized problem, that the Lorentz force acted to enhance the helicity of the convective columns at the onset of convection (characterized by a columnar flow aligned with the axis of rotation, as illustrated in the figure by the title). While helicity (the dot product of velocity and 
(a)

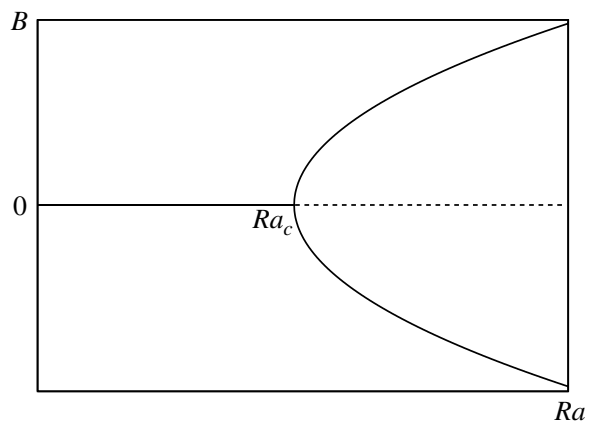

(b)

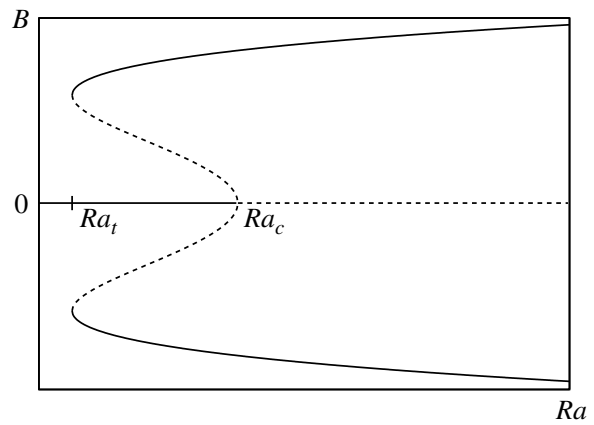

FIGURE 1. Two bifurcation diagrams often observed for dynamo simulations. The left diagram presents a typical supercritical bifurcation, whereas the right diagram exemplifies a subcritical bifurcation (in both cases $B$ and $-B$ are solutions). Solid lines indicate stable branches, and dashed lines unstable branches. The subcritical bifurcation is characterized by a turning point $\left(R a=R a_{t}\right)$. While on the right diagram the magnetic field destabilizes from the purely hydrodynamic (non-magnetic) solution when $R a=R a_{c}$, the dynamo solution can be maintained down to this turning point, resulting in a hysteretic behaviour.

vorticity) is not a necessary ingredient for dynamo action, it is known to help magnetic field amplification. Sreenivasan \& Jones (2011) therefore point to a mechanism by which the leading effect of the Lorentz force, rather than saturating the magnetic field, destabilizes it. They furthermore highlight that this effect is dominant for a dipolar axial magnetic field, which could explain why these configurations (reminiscent of the Earth's magnetic field) are so often observed numerically.

Subcritical dynamos are characterized by a turning point for $R a=R a_{t}$ below which dynamo action is lost. For $R a \in\left[R a_{t}, R a_{c}\right]$ two different solutions can be observed, either the purely hydrodynamic solution or a dynamo branch. This will depend on the initial condition (both in terms of geometry and amplitude), which will lie in the basin of attraction of one solution or the other. Another striking feature of a subcritical dynamo is the catastrophic collapse of the magnetic field observed when the control parameter $R a$ is decreased from values slightly above $R a_{t}$ to values slightly less than $R a_{t}$. This mechanism could provide a possible explanation for the sudden collapse of the Martian dynamo (Kuang et al. 2008).

\section{Future}

How dynamo action takes place is a delicate issue. The bifurcation can be either supercritical or subcritical, depending on the parameters. The role of the Ekman and magnetic Prandtl numbers in the scenario introduced by Sreenivasan \& Jones (2011) remains to be understood. Even more surprising diagrams have been observed for dynamo action. In some cases the dynamo branch of solutions does not connect to the purely hydrodynamic mode at all, but consists of a detached lobe of solution (Morin 2005; Morin \& Dormy 2009) known as an isola. Isola solutions are associated with configurations for which the purely hydrodynamic solution does not destabilize. A finite amplitude initial field is then always needed to achieve dynamo action, and the dynamo branch is bounded by two turning points at small and large values of the control parameter. Such diagrams were also obtained with earlier reduced (mean-field) models (see Soward \& Jones 1983). The transition between these three types of 
bifurcation diagrams, and the role of the Ekman and magnetic Prandtl numbers, surely deserve further study.

Historically, another mechanism for subcriticality was anticipated for the geodynamo. Early numerical and theoretical work (see Roberts 1988) suggested that the Lorentz force could relax the strong constraint imposed by the rapid rotation of the reference frame and thus lower the critical Rayleigh number for the onset of convection. Both magnetic effects and rotation effects taken separately impede convection, but combined they can partly cancel each other and yield a lower threshold. This bifurcation diagram is characterized by a first supercritical bifurcation to a so-called 'weak-field' branch, followed (as the control parameter is increased) by a runaway field growth (turning point) to the 'strong field' branch, on which the Lorentz force is of comparable strength to the Coriolis force. Once on this branch, it is anticipated that dynamo action can be maintained at values of the Rayleigh number even lower than the critical value for the onset of convection. This scenario relies on a different mechanism to that invoked by Sreenivasan \& Jones (2011) and both effects may in fact take place in numerical models depending on the parameter regime. Other hysteretic transitions have been reported at larger values of the control parameter. As the Rayleigh number is further increased, dipolar dynamos destabilize towards multipolar reversing dynamos (Kutzner \& Christensen 2002). This latter transition can be hysteretic (Simitev \& Busse 2009). Further studies along the line of the work of Sreenivasan \& Jones (2011), i.e. combining numerical and analytical work, will be necessary before one can claim to have unravelled the mystery of the origin of the Earth's magnetic field.

\section{References}

Busse, F. H. 1970 Thermal instabilities in rapidly rotating systems. J. Fluid Mech. 44, 441-460.

Christensen, U., Olson, P. \& Glatzmaier, G. A. 1999 Numerical modelling of the geodynamo: a systematic parameter study. Geophys. J. Intl 138, 393-409.

Dormy, E., Soward, A. M., Jones, C. A., Jault, D. \& Cardin, P. 2004 The onset of thermal convection in rotating spherical shells. J. Fluid Mech. 501, 43-70.

Jones, C. A., Soward, A. M. \& Mussa, A. I. 2000 The onset of thermal convection in a rapidly rotating sphere. J. Fluid Mech. 405, 157-179.

KuAnG, W., JiAng, W. \& WAng, T. 2008 Sudden termination of Martian dynamo: implications from subcritical dynamo simulations. Geophys. Res. Lett. 35, L14204.

Kutzner, C. \& Christensen, U. 2002 From stable dipolar towards reversing numerical dynamos. Phys. Earth Planet. Inter. 131, 29-45.

MoRIN, V. 2005 Instabilités et bifurcations associées à la modélisation de la Géodynamo. PhD thesis, Université Paris VII http://tel.archives-ouvertes.fr/tel-00011484/en/.

Morin, V. \& Dormy, E. 2009 The dynamo bifurcation in rotating spherical shells. Intl J. Mod. Phys. B 23 (28-29), 5467-5482.

Roberts, P. H. 1965 On the thermal instability of a highly rotating fluid sphere. Astrophys. J. 141 (1), 240-250.

RoBerTS, P. H. 1968 On the thermal instability of a rotating-fluid sphere containing heat sources. Phil. Trans. R. Soc. Lond. A 263, 93-117.

Roberts, P. H. 1988 Future of geodynamo theory. Geophys. Astrophys. Fluid Dyn. 44, 3-31.

SimiteV, R. D. \& BUSSE, F. H. 2009 Bistability and hysteresis of dipolar dynamos generated by turbulent convection in rotating spherical shells. Earth Planet. Sci. Lett. 85, 19001.

SowArd, A. M. \& Jones, C. A. $1983 \alpha^{2}$-Dynamos and Taylor's constraint. Geophys. Astrophys. Fluid Dyn. 27, 87-122.

SREEnivasAn, B. \& JONES, C. A. 2011 Helicity generation and subcritical behaviour in rapidly rotating dynamos. J. Fluid Mech. 688, 5-30.

YANO, J.-I. 1992 Asymptotic theory of thermal convection in rapidly rotating systems. J. Fluid Mech. 243, 103-131. 